\title{
A Tug of War: DNA-Sensing Antiviral Innate Immunity and Herpes Simplex Virus Type I Infection
}

\author{
Yingying Lin ${ }^{1}$ and Chunfu Zheng ${ }^{1,2 *}$ \\ ${ }^{1}$ Department of Immunology, School of Basic Medical Sciences, Fujian Medical University, Fuzhou, China, ${ }^{2}$ Department of \\ Microbiology, Immunology and Infectious Diseases, University of Calgary, Calgary, AB, Canada
}

Cytosolic DNA sensors are the most recently described class of pattern recognition receptors (PRRs), which induce the production of type I interferons (IFN-I) and trigger the induction of a rapid and efficient innate immune response. Herpes simplex virus type I (HSV-1), a typical DNA virus, has displayed the ability to manipulate and evade host antiviral innate immune responses. Therefore, with an aim to highlight IFN-I-mediated innate immune response in a battle against viral infection, we have summarized the current understandings of DNA-sensing signal pathways and the most recent findings on the molecular mechanisms utilized by HSV-1 to counteract antiviral immune responses. A comprehensive understanding

OPEN ACCESS

Edited by:

Soren R. Paludan,

Aarhus University, Denmark

Reviewed by:

Malathi Krishnamurthy, University of Toledo, United States

Junji Xing,

Houston Methodist Research Institute, United States

*Correspondence: Chunfu Zheng

zheng.alan@hotmail.com

Specialty section: This article was submitted to Virology,

a section of the journal

Frontiers in Microbiology

Received: 28 July 2019

Accepted: 29 October 2019

Published: 26 November 2019

Citation:

Lin Y and Zheng C (2019) A Tug of War: DNA-Sensing Antiviral Innate Immunity and Herpes Simplex Virus

Type I Infection.

Front. Microbiol. 10:2627.

doi: 10.3389/fmicb.2019.02627 of the interplay between HSV-1 and host early antiviral immune responses will contribute to the development of novel therapies and vaccines in the future.

Keywords: herpes simplex virus type I, DNA sensor, immune evasion, interferon, antiviral immunity

\section{INTRODUCTION}

Herpes simplex virus type I (HSV-1), a member of the alphaherpesvirus subfamily, has already co-evolved with human beings for thousands of years and is well known for its high prevalence in the population worldwide (Davison, 2010; Sharma et al., 2016; Ibanez et al., 2018). With a large linear double-stranded DNA that encodes over 80 proteins, HSV-1 can produce lifelong infections in the host, and this is achieved thanks to its capacity to infect epithelial cells, neurons, and other cell types, including immune cells in vivo and in vitro (Wu et al., 2016; Koelle et al., 2017; Tognarelli et al., 2019).

Clinically, HSV-1 is mainly associated with orofacial lesions, yet it is also the leading cause of infectious blindness in developed countries and viral encephalitis in adults (Horowitz et al., 2010; Farooq and Shukla, 2012; Bernstein et al., 2013). After the initial infection, HSV-1 becomes latent in the trigeminal ganglion, and recurrent reactivation leads to different immunopathology, which may cause neuronal damage and Alzheimer's disease (AD) (Devanand, 2018).

Early detection of viral invasion by pattern recognition receptors (PRRs) is crucial for the induction of a rapid and efficient innate immune response. Cytosolic DNA sensors are the most recently described class of PRRs, which also include the Toll-like receptors (TLRs), certain RNA sensors, such as RIG-I-like receptors and melanoma differentiation-associated gene 5 (Wu and Chen, 2014; Su et al., 2016). Viral nucleic acids of HSV-1, recognized by various PRRs, can act as strong activators of various signaling pathways that finally promote antiviral immune responses through the secretion of pro-inflammatory cytokines, as well as the production of type-I interferons (IFN-I) in infected cells (Iwasaki, 2012). The activation 
of the IFN-I pathway ultimately induces the expression of multiple IFN-stimulated genes (ISGs) and boosts the innate immune responses (Schoggins, 2019). HSV-1 has been reported to evade host immunity and facilitate its infection and replication through multiple strategies (Schulz and Mossman, 2016; Christensen and Paludan, 2017).

Although different cytosolic DNA-sensing pathways can be activated, HSV-1 has developed multiple mechanisms to attenuate this host antiviral machinery (Zheng, 2018). In this review, we outline the recent findings with the aim of highlighting antiviral innate immune responses in the battle against the HSV infection. A comprehensive understanding of the interplay between HSV-1 and host antiviral innate immunity could contribute to the development of novel immunotherapies and effective vaccines to counteract this virus over the next few decades.

\section{INTERPLAY BETWEEN THE HOST ANTIVIRAL DNA-SENSING PATHWAYS AND HERPES SIMPLEX VIRUS TYPE I}

The newly emerging DNA in the cytoplasm induces robust and rapid innate immune responses through its binding to various DNA sensors, including TLR9, absent in melanoma 2 (AIM2), RNA polymerase III, Interferon- $\gamma$ inducible protein 16 (IFI16), DEAD-box helicase 41 (DDX41), and some proteins involved in the DNA damage responses, among which the cyclic guanosine monophosphate-adenosine monophosphate synthase (cGAS) is the only one that has been identified as a universal cytoplasmic DNA sensor in various cell types (Lund et al., 2003; Chiu et al., 2009a; Zhang et al., 2011; Sun et al., 2013; Zheng, 2018; Stempel et al., 2019). TLRs have been described to mediate antiviral activities against HSV during infection. If the animals lacked both TLR2 and TLR9, all animals were more susceptible to infection than single knockout animals pointing out the relevance of these receptors during HSV infection (Lima et al., 2010; Uyangaa et al., 2018). Furthermore, HSV-1 infection in human neurons was shown to be suppressed by type-III IFN (IFN- $\lambda$ ) through the upregulation of TLR9 expression and subsequent TLR9-mediated antiviral responses involving the transcription factor interferon regulatory factor 7 (IRF7) (Zhou et al., 2011). But this result remains to be determined because IFN- $\lambda$ has been reported to be secreted during HSV infection in the vaginal mucosa, mainly by dendritic cells (Iversen et al., 2010). Although AIM2 also detects aberrantly localized DNA, it is currently proposed that it cooperates with IFI16 and activates the inflammasome (Lugrin and Martinon, 2018). Other proposed DNA sensors, such as DDX41, also require further investigation to clarify their role during HSV infection and if they act redundantly in a cell-type-dependent manner (Zhang et al., 2011). Furthermore, unlike cGAS and IFI16, these sensors have, thus far, not been shown to restrict the replication of HSV-1 and have been evaded by HSV-1.

In this review, specific attention is given to the cGASSTING DNA-sensing signal pathways and its downstream IFN-I signal pathway, which plays a central role in innate antiviral immunity (Figure 1).

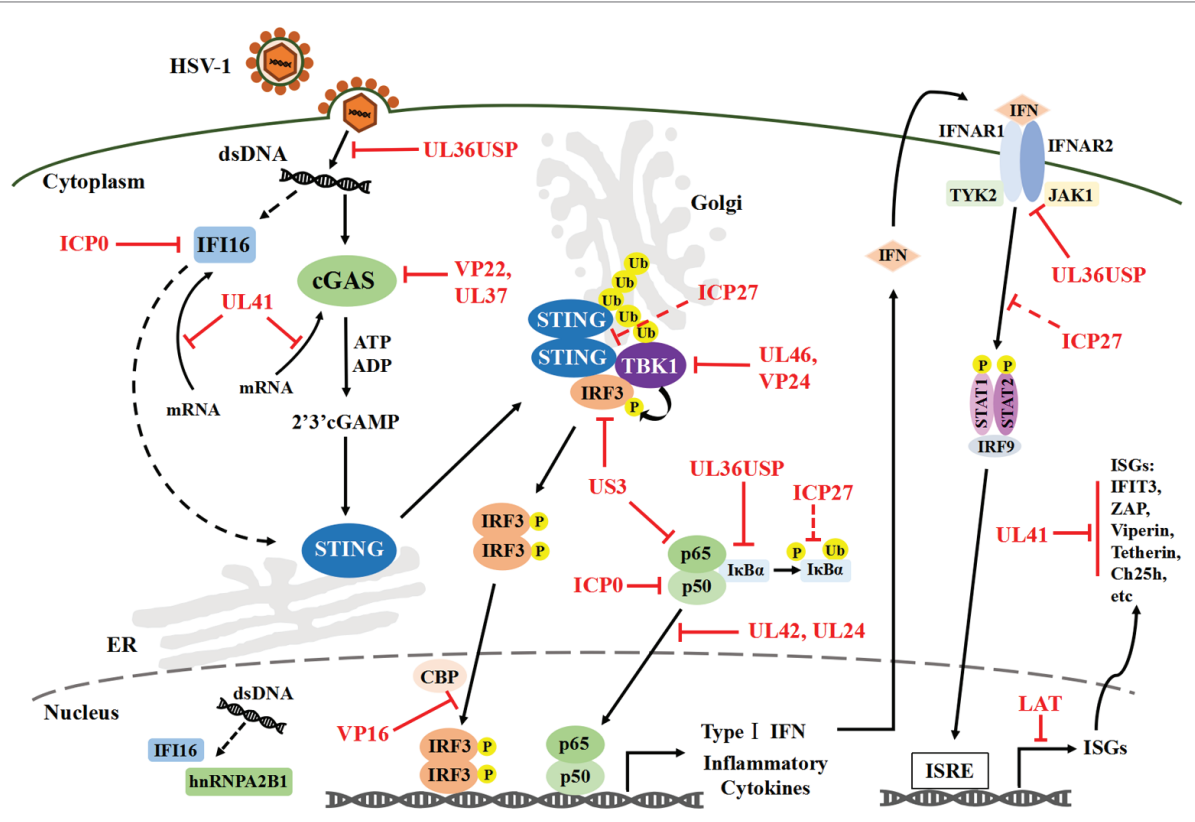

FIGURE 1 | HSV-1 mediated evasion of the DNA-sensing pathway in innate immunity. Cytosolic DNA sensors, such as IFI16 and cGAS, can recognize HSV-1 dsDNA and trigger IRF3 and NF-kB activation, which results in the production of IFN-I and antiviral immune responses. Multiple steps in the DNA-sensor-mediated IFN-I signaling pathway can be targeted by HSV-1 proteins, including cGAS-mediated viral recognition and subsequent signaling pathways. Solid lines indicate confirmed interactions between the host signaling proteins and HSV-1 proteins. Dashed lines indicate uncertain interactions. HSV-1, Herpes simplex virus type I; IFI16, Interferon- $\gamma$ inducible protein 16; cGAS, Cyclic guanosine monophosphate-adenosine monophosphate synthase; dsDNA, Double-stranded DNA; IRF3, Interferon regulatory factor 3; NF-кB, Nuclear factor kB; IFN-I, Type I interferons; P, Phosphate; Ub, Ubiquitin. 


\section{Interferon- $\gamma$ Inducible Protein 16}

IFI16 is a host sensor of nucleic acids that has been reported to recognize cytosolic double-stranded DNA (dsDNA) as well as HSV-1-derived DNA in the nucleus (Unterholzner et al., 2010). HSV-1 recognition by IFI16, which itself results in acetylation and redistribution to the cytoplasm, then induces the activation of the transcription factor interferon regulatory factor 3 (IRF3) and transcription factor's nuclear factor $\kappa \mathrm{B}$ (NF- $\kappa B$ ) into the nucleus (Pilli et al., 2012; Ansari et al., 2015). These processes are followed by the production of IFN-I and IL-6, which are able to restrict viral replication and initiate an inflammatory response (Conrady et al., 2012; Ansari et al., 2015; Zheng, 2018).

Furthermore, the host sensor IFI16 (or AIM2) that encounters viral determinants could also interact with the inflammasome and hence induce the pro-caspase- 1 activation by an apoptosisassociated speck-like protein containing a caspase recruitment domain (Johnson et al., 2013). HSV-1 immediate early protein infected-cell polypeptide 0 (ICP0), which is an E3 ubiquitin ligase, has been shown to successfully inhibit IFI16 activation by guiding it to the proteasome and accelerating its degradation (Johnson et al., 2013). HSV viral protein 22 (VP22), encoded by the UL49 gene, has also been reported to block this pro-caspase-1 activation and inhibit the secretion of pro-inflammatory cytokines such as IL-1 $\beta$ or IL-8 (Maruzuru et al., 2018). Moreover, apart from directly inhibiting the activation of IFI16, a recent study revealed that an HSV-1 UL41 protein contributed to the decrease of IFI16 expression by degrading its mRNA (Orzalli et al., 2016).

In addition, within the nucleus, viral DNA is not only sensed but also loaded with heterochromatin to silence its expression and to restrict viral replication (Orzalli and Knipe, 2014). Several restriction factors, including IFI16 and promyelocytic leukemia (PML) protein, limit viral gene expression and replication (Orzalli et al., 2013; Merkl et al., 2018). Merkal et al. have defined a novel mechanism of epigenetic silencing of HSV-1 DNA, which revealed that an IFI16 filamentous structure could recruit other restriction factors, including PML protein, speckled protein 100 (Sp100), and alpha-thalassemia mental retardation syndrome $\mathrm{x}$-linked (ATRX), to aid in the restriction (Merkl and Knipe, 2019). Notably, an increasing body of evidence suggests that HSV-1 ICP0 protein can promote the degradation of the IFI16, ATRX, Sp100, as well as PML proteins and prevent their restriction activities (Lukashchuk and Everett, 2010; Jurak et al., 2012; Orzalli et al., 2012). However, further studies are still needed to identify the full protein composition of this new infected cell nuclear structure and investigate the underlying mechanisms by which HSV-1 regulates the downstream pathways related to IFI16.

\section{Cyclic Guanosine Monophosphate- Adenosine Monophosphate Synthase- Stimulator of Interferon Genes}

Several DNA viruses, including adenovirus, cytomegalovirus, hepatitis B virus, and HSV-1, can induce IFN-I in a cGAS/ stimulator of interferon genes (STING)-dependent pathway (Gao et al., 2013; Ma et al., 2015; Wu et al., 2015; Dansako et al., 2016; Paijo et al., 2016). As a result, cGAS plays a crucial role in antiviral innate immunity, which triggers cyclic GMP-AMP (cGAMP) production through its enzymatic activity upon binding to cytosolic dsDNA (Cai et al., 2014). Then, cGAMP activates the endoplasmic reticulum (ER)-anchored STING, which then translocates from the ER to the Golgi apparatus and leads to the recruitment and phosphorylation of TANK-binding kinase 1 (TBK1) and I $\mathrm{KB}$ kinase (IKK). Notably, the trafficking step from the ER to the Golgi apparatus is crucial for the induction of IFN-I transcription by STING (Saitoh et al., 2009). Finally, the IRF3 and NF- $\kappa$ B are activated to induce the production of IFN-I and inflammatory cytokines (Sun et al., 2013; Wu et al., 2013).

At the upstream of the cGAS/STING signaling pathway, our previous study showed that an HSV-1 UL41 protein could degrade cGAS mRNA during viral infection ( $\mathrm{Su}$ and Zheng, 2017). This finding showed that the ectopic expression of UL41 remarkably reduced the accumulation of cGAS via its RNase activity and downregulated the cGAS/STING-mediated activation of the IFN pathway to escape antiviral innate immune responses. Besides, HSV-1VP22, a highly abundant tegument protein, also has an effect on this DNA-sensing pathway. VP22 has been shown to interact directly with cGAS and thus suppress the enzymatic activity of cGAS, and it acts as an important inhibitor of IFN- $\beta$ production and downstream antiviral genes (Huang et al., 2018).

Interestingly, Deschamps et al. have showed that the stable overexpression of HSV-1 tegument protein UL46 in cells can reduce the expression of STING and inhibit its downstream IFN-I signaling pathway (Deschamps and Kalamvoki, 2017). However, during viral infection, UL46 does not affect the expression and function of STING, which is obviously illogical. Our recent study revealed a novel underlying mechanism that HSV-1 UL46 downregulated antiviral immune responses by interacting directly with TBK1. UL46 was shown to significantly reduce the dimerization of TBK1 and affect the interaction between TBK1 and IRF3, which resulted in inhibiting the activation of TBK1 and the production of IFN- $\beta$ (You et al., 2019). Based on this evidence, HSV-1 UL46 disrupts the cGAS-STING signaling pathway and possibly interacts with both STING and TBK1 via separate domains.

An increasing amount of evidence has shown that posttranslational modifications, such as phosphorylation and ubiquitination, directly or indirectly modulate the cGAS/STING pathway and significantly affect viral infections (Liu et al., 2013, 2016). cGAS can be targeted for deamidation by the HSV-1 tegument protein UL37, which causes cGAS inactivation and facilitates HSV-1 lytic replication (Zhang et al., 2018). Moreover, Sun et al. demonstrated that HSV-1 UL36 ubiquitin-specific protease (UL36USP) could inhibit viral capsid ubiquitination and subsequent degradation through its deubiquitylase activity, thus avoiding the recognition of cGAS instead of affecting the stability of cGAS or STING (Sun et al., 2015).

These findings reveal some novel mechanisms of viral evasion. More importantly, the multifaceted strategy of HSV-1 to 
compromise the DNA-sensing pathway highlights how STING is a key restriction factor for HSV-1.

\section{TANK-Binding Kinase 1-Interferon Regulatory Factor 3}

At the downstream of the cGAS/STING signaling pathway, studies from our lab demonstrated that VP24, a serine protease of HSV-1, could also block dsDNA-triggered IFN production by abrogating the interaction between TBK1 and IRF3 and inhibiting the activation of IRF3 (Zhang et al., 2016), while HSV-1 VP16 could prevent IRF3 from recruiting the CREB-binding protein (CBP) coactivator, thus blocking IRF3-mediated transcription (Xing et al., 2013). What is more, protein kinase US3 of HSV-1 has been shown to interact with and hyperphosphorylate IRF3 at Ser175 to prevent IRF3 activation and dampen IFN-I production (Wang et al., 2013, 2014). Christensen et al., found that HSV-1 ICP27 protein, a product of viral immediate early genes conserved among all human herpesviruses, could impair the upstream of IRF3 activation (but could not impair TBK1 phosphorylation) by interacting with TBK1 and STING in human macrophages (Christensen et al., 2016). Nevertheless, ICP27, as an immediate early gene, can regulate the production of many viral genes through stimulating transcription and translation of viral early and late genes, indicating that the results from the ICP27 deletion virus does not guarantee that the viral immune evasion is mediated by ICP27 (Sandri-Goldin, 2011). It is plausible that ICP27 might affect the IFN-I signaling pathway through the regulation of the expression of viral early and late genes. Altogether, these findings from our and other labs will be important for understanding the interaction between HSV-1 and the host DNA-sensing signal pathway.

\section{TANK-Binding Kinase 1-Nuclear Factor $\kappa B$}

$\mathrm{NF}-\kappa \mathrm{B}$ is known for its critical role in innate immune responses and can be strongly induced at the downstream of most PRRs, resulting in the production of IFN- $\beta$ as well as inflammatory interleukins (Woronicz et al., 1997). During the activation of NF- $\kappa B$, IкBs are phosphorylated by activated $\mathrm{IKK}$, and then the NF-KB p50/p65 heterodimer is released and transferred to the nucleus, which finally regulates the innate immune responses (Bonizzi and Karin, 2004; Hayden and Ghosh, 2008; Chiu et al., 2009b; Iwai, 2014). New evidence suggests that TBK1 is essential for the activation of the NF- $\mathrm{KB}$ signaling pathway mediated by dsDNA and utilizes the IKK activation loop to activate the subunit p65 (Abe and Barber, 2014).

Consequently, HSV-1 has also evolved various elaborate mechanisms to subvert this signaling pathway. For example, the HSV-1 immediate early protein ICP0 interacts with NF- $\mathrm{KB}$ subunits p50/p65 and degrades p50 through its E3-ubiquitin ligase activity (Zhang et al., 2013a). Kim et al. also reported that HSV-1 ICP27 could repress NF-kB activity through blocking the phosphorylation and ubiquitination of ІкB $\alpha$ and stabilize I $B \alpha$ to evade immune responses during the very early period of HSV-1 infection (Kim et al., 2008). Meanwhile, our study has showed that HSV-1 UL36USP deubiquitinates IKB $\alpha$ and prevents its degradation, which inhibits p50/p65 transportation and finally abrogates NF- $\mathrm{KB}$ activation (Ye et al., 2017).

The production of IFN-I depends on transcription factors of both IRF3 and NF- $\mathrm{KB}$, which bind to distinct regulatory domains in the promoter. HSV-1 US3 has been shown to hyperphosphorylate p65/RelA at serine 75 , which significantly inhibited NF- $\mathrm{kB}$ activation by blocking its nuclear translocation and decreased the expression of inflammatory chemokine interleukin-8 (Wang et al., 2013, 2014). Similarly, HSV-1 UL42, a DNA polymerase processivity factor, also significantly prevents NF- $\mathrm{KB}$-dependent gene expression by retaining p50/p65 in the cytoplasm (Zhang et al., 2013b). Additionally, HSV-1 UL24, another conserved viral protein that is important for viral replication, selectively blocked activation of the NF- $\mathrm{\kappa B}$, but not IRF3, by binding to Rel homology domains of p50/p65 and abrogating their nuclear translocation (Xu et al., 2017). For the first time, UL42 and UL24 are demonstrated to effectively inhibit cGAS/STING-induced NF- $\mathrm{kB}$ activation and dsDNAmediated IFN- $\beta$ or IL- 6 production during HSV-1 infection. It is worth noting that some HSV-1 proteins may target the cytosolic DNA-sensing pathway through similar mechanisms.

\section{Janus Kinase-Signal Transducer and Activator of Transcription}

Although HSV-1 can antagonize the production of IFN-I via many mechanisms, a certain amount of IFN-I produced during early infection will induce ISGs through the Janus kinase-signal transducer and activator of transcription (JAK-STAT). HSV-1 also evolves mechanisms to disrupt the JAK-STAT pathway, which is the downstream of the IFN signaling pathway, and further evades the antiviral immunity.

It is known that IFN-I can be initially produced following the detection of viral RNA, DNA, or proteins by intracellular PRRs in host cells (Raftery and Stevenson, 2017). IFN-I include IFN- $\alpha$ as well as IFN- $\beta$, IFN- $\varepsilon$, IFN- $\kappa$, and IFN- $\omega$ (van Pesch et al., 2004; Ivashkiv and Donlin, 2014). After its secretion, IFN-I interacts with the cell-surface receptor known as the type I IFN receptor (IFNAR), which is a heteromeric receptor that contains subunit IFNAR1 and IFNAR2 (Decker et al., 2005). When the receptor is activated, it recruits and phosphorylates the tyrosine kinase 2 (Tyk2) and JAK1, which leads to the formation of a heterodimer of phosphorylated STAT1 and STAT2 (Brooks et al., 2014). Then, STAT1/STAT2 binds to the cytoplasmic IRF9, forming a complex known as IFN-stimulated gene factor 3 (ISGF3) that translocates into the nucleus and binds to a DNA sequence called the IFN-stimulated response element (ISRE), resulting in the transcription of many ISGs, including viperin, zinc-finger antiviral protein (ZAP), Cholesterol 25-hydroxylase (Ch25h), tetherin, ISG15, and some proteins of the tripartite motif (TRIM) family, which are responsible for the effector properties of directly antiviral responses (Schoggins and Rice, 2011; Schneider et al., 2014; Raftery and Stevenson, 2017). However, HSV-1 has evolved multiple strategies to evade this process.

Johnson et al. observed that HSV-1 protein ICP27 was sufficient to inhibit IFN-mediated STAT1 phosphorylation and 
nuclear accumulation at or before the phosphorylation of JAK (Johnson et al., 2008; Johnson and Knipe, 2010). HSV-1 can also downregulate the protein levels of JAK1 and STAT2 through the virion host shutoff protein at a relatively high multiplicity of infection (Chee and Roizman, 2004). Previous studies from our and other labs have shown that UL41 can degrade the mRNAs of some ISGs, such as IFIT3, ZAP, viperin, tetherin, as well as Ch25h, to attenuate the IFN-mediated antiviral immune responses via its RNase activity (Zenner et al., 2013; Shen et al., 2014; Su et al., 2015; Jiang et al., 2016; You et al., 2017; Yuan et al., 2018). Furthermore, we have recently demonstrated that HSV-1 UL36USP also antagonizes the activation of the IFN-JAK-STAT pathway through specifically binding to IFNAR2 and blocking the interaction between JAK1 and IFNAR2, which is independent of its DUB activity (Yuan et al., 2018).

The HSV-1 latency-associated transcript (LAT), which is not known to encode a functional protein but regulate the virus latency and reactivation, has been shown to inhibit apoptosis via inhibiting activation of pro-apoptotic caspases and promoting cell survival or immune evasion (Perng et al., 2000; Henderson et al., 2002; da Silva and Jones, 2013; Phelan et al., 2017). However, the mechanism of this process is unknown. Tormanen et al. have recently observed that LAT affected apoptosis by downregulating the expression of JAK1 and JAK2, as well as several downstream ISGs of the JAK-STAT pathway at the level of a transcriptional mechanism during HSV-1 latency (Tormanen et al., 2019).

Overall, a growing amount of evidence suggests that HSV-1 has evolved multiple mechanisms to inhibit IFN signaling not only in infected cells but also in neighboring cells, thereby allowing for increased viral replication and spread. Therefore, the increased understanding of the IFN-JAK-STAT signal pathway is essential for our ambition to develop novel, less toxic, and more effective anti-viral treatments.

\section{VIRAL MANIPULATION OF OTHER ANTIVIRAL PROCESSES VIA REGULATING TRIPARTITE MOTIF PROTEINS}

The function of autophagy is well known for its regular degradation and recycling of cellular components through isolating certain targeted cytoplasmic proteins within a doublemembraned autophagosome (Dong and Levine, 2013). The pathway of autophagy is an essential component of host defense against viral infection and innate immune responses (Lussignol and Esclatine, 2017). Besides, recent studies have demonstrated that autophagy and innate immune signaling, in particular the IFN-I signaling pathway, are intricately interconnected (Tal and Iwasaki, 2009; Levine et al., 2011; Deretic et al., 2013). It is worth noting that several key molecules, such as TBK1, IRF3, and $\mathrm{p} 62$, involved in IFN- $\beta$ induction are also important regulators of autophagy (Sharma et al., 2003; Pilli et al., 2012; Richter et al., 2016).
Interestingly, TRIM proteins, which belong to the larger family of RING E3 ligases and are well known to regulate antiviral cytokine production in DNA-sensing pathways, also play important roles in autophagy as well as autophagy-mediated antiviral defenses (Versteeg et al., 2013; Mandell et al., 2014). Previous study indicated that HSV-1 infection could lead to ER stress-relating signaling networks including many pathways of immune responses and other mechanisms that restrict viral pathogenesis (Li et al., 2015). However, to survive and propagate within the host, many viruses, including HSV-1, have evolved a variety of strategies to evade autophagy for their own benefit.

It has been proved that both TRIM56 and TRIM32 could catalyze K63-linked polyubiquitination on STING when STING had been activated by cGAMP and then translocated from the ER to the Golgi apparatus (Tsuchida et al., 2010; Ishikawa and Barber, 2011; Zhang et al., 2012). Meanwhile, after activation of TBK1 and IRF3, this excess of STING can be degraded by p62/Sequestosome1-dependent autophagy (Prabakaran et al., 2018). Konstantin et al. have revealed that unconventional K27-linked auto-ubiquitination is essential for the GTP hydrolysis activity of TRIM23, which is necessary for the recruitment of TRIM23 to autophagosomal membranes and the activation of TBK1- and p62-mediated selective autophagy (Sparrer et al., 2017). Interestingly, HSV-1 US11 could drastically suppress this autophagy loop by disrupting the TRIM23-TBK1-Hsp90 complex and inhibiting the restriction of HSV-1 infection (Liu et al., 2018). The underlying mechanism is that US11 can block recruitment of TBK1 by targeting the C-terminal ADP-ribosylation factor domain in TRIM23, which results in a negative impact on both pathways of autophagy and the type I IFN response.

Many functions of HSV-1 ICP0 have been directly linked to its E3 ubiquitin ligase activity that is required for efficient infection (Lilley et al., 2010; Orzalli et al., 2012, 2013). Conwell et al. have presented that ICP0 utilized its own RING E3 ligase activity to induce polyubiquitination and degradation of TRIM27, which might play a role in intrinsic resistance to HSV-1 infection (Conwell et al., 2015). Similarly, the Epstein-Barr virus induces the expression of TRIM29, which was reported to modify STING with K48-linked polyubiquitin and negatively regulate innate immune responses to DNA viruses (Xing et al., 2017).

The results revealed some previously undocumented mechanisms of DNA viruses in infected cells and their resistance to innate immunity, which has greatly improved our understanding of the interplay between HSV-1 and host antiviral responses through targeting TRIM family.

\section{CONCLUSION AND PERSPECTIVES}

In this review, a growing number of findings have explained the active interactions between HSV-1 and the host antiviral innate immunity, which have revealed some novel mechanisms of viral evasion. Upon infection, HSV-1 has developed sophisticated strategies with viral proteins to counteract IFN-I production in innate immune responses, mainly through interactions with the DNA-sensor-mediated antiviral signal pathways. Through these 
achievements, we stand to gain an enriched understanding of viral evasion mechanisms in host cells. Nevertheless, there remain several knowledge gaps to be further investigated.

Firstly, how cGAS senses and binds to the dsDNA of HSV-1 has remained elusive. Secondly, the mechanisms through which the dysregulation of innate immune responses by HSV-1 affect human viral diseases and pathogenesis, such as $\mathrm{AD}$, remain largely elusive. In other words, the clinical models of many observations coming from the overexpression of viral proteins in human cell systems remain to be established in the future. Thirdly, it warrants further investigation whether HSV-1 evades the antiviral potential of the TRIM family, and this will open up a new potential area of viral immune escape mechanisms.

Strikingly, there is still a firestorm of controversy about what is the DNA sensor of a virus in the nucleus really is. Finally, perhaps most challenging and essential issue is what nuclear receptor initiates the innate immune response to DNA viruses, how does it achieve this, and does it include HSV-1? Surprisingly, during preparation of our manuscript, a recent discovery from Cao's group has showed that the nuclear-localized heterogeneous nuclear ribonucleoprotein A2B1 (hnRNPA2B1) recognizes viral DNA and then translocates to the cytoplasm where it activates

\section{REFERENCES}

Abe, T., and Barber, G. N. (2014). Cytosolic-DNA-mediated, STING-dependent proinflammatory gene induction necessitates canonical NF-kappaB activation through TBK1. J. Virol. 88, 5328-5341. doi: 10.1128/JVI.00037-14

Ansari, M. A., Dutta, S., Veettil, M. V., Dutta, D., Iqbal, J., Kumar, B., et al. (2015). Herpesvirus genome recognition induced acetylation of nuclear IFI16 is essential for its cytoplasmic translocation, Inflammasome and IFN-beta responses. PLoS Pathog. 11:e1005019. doi: 10.1371/journal.ppat.1005019

Bernstein, D. I., Bellamy, A. R., Hook, E. W. 3rd, Levin, M. J., Wald, A., Ewell, M. G., et al. (2013). Epidemiology, clinical presentation, and antibody response to primary infection with herpes simplex virus type 1 and type 2 in young women. Clin. Infect. Dis. 56, 344-351. doi: 10.1093/cid/cis891

Bonizzi, G., and Karin, M. (2004). The two NF-kappaB activation pathways and their role in innate and adaptive immunity. Trends Immunol. 25, 280-288. doi: 10.1016/j.it.2004.03.008

Brooks, A. J., Dai, W., O'Mara, M. L., Abankwa, D., Chhabra, Y., Pelekanos, R. A., et al. (2014). Mechanism of activation of protein kinase JAK2 by the growth hormone receptor. Science 344:1249783. doi: 10.1126/science.1249783

Cai, X., Chiu, Y. H., and Chen, Z. J. (2014). The cGAS-cGAMP-STING pathway of cytosolic DNA sensing and signaling. Mol. Cell 54, 289-296. doi: 10.1016/j. molcel.2014.03.040

Chee, A. V., and Roizman, B. (2004). Herpes simplex virus 1 gene products occlude the interferon signaling pathway at multiple sites. J. Virol. 78, 4185-4196. doi: 10.1128/JVI.78.8.4185-4196.2004

Chiu, Y. H., Macmillan, J. B., and Chen, Z. J. (2009a). RNA polymerase III detects cytosolic DNA and induces type I interferons through the RIG-I pathway. Cell 138, 576-591. doi: 10.1016/j.cell.2009.06.015

Chiu, Y. H., Zhao, M., and Chen, Z. J. (2009b). Ubiquitin in NF-kappaB signaling. Chem. Rev. 109, 1549-1560. doi: 10.1021/cr800554j

Christensen, M. H., Jensen, S. B., Miettinen, J. J., Luecke, S., Prabakaran, T., Reinert, L. S., et al. (2016). HSV-1 ICP27 targets the TBK1-activated STING signalsome to inhibit virus-induced type I IFN expression. EMBO J. 35, 1385-1399. doi: 10.15252/embj.201593458

Christensen, M. H., and Paludan, S. R. (2017). Viral evasion of DNA-stimulated innate immune responses. Cell. Mol. Immunol. 14, 4-13. doi: 10.1038/cmi.2016.06

Conrady, C. D., Zheng, M., Fitzgerald, K. A., Liu, C., and Carr, D. J. (2012). Resistance to HSV-1 infection in the epithelium resides with the novel innate sensor, IFI-16. Mucosal Immunol. 5, 173-183. doi: 10.1038/mi.2011.63 the TBK1-IRF3 pathway and amplifies IFN- $\alpha / \beta$ production (Wang et al., 2019). But, whether hnRNPA2B1 plays an important role in initiating the IFN production and enhancing the cytoplasmic antiviral signaling in HSV-1 infection still needs further investigation. Further understanding of these questions will help us to reveal the detailed and molecular mechanisms of HSV-1 infection or viral diseases, which may accelerate future development of novel antiviral therapeutics and vaccines.

\section{AUTHOR CONTRIBUTIONS}

YL wrote the manuscript and designed the figures. CZ reviewed and modified the manuscript.

\section{FUNDING}

This article was supported by grants from the National Natural Science Foundation of China (No. 81602498), the Natural Science Foundation of Fujian Province (No. 2018J01830), and the MiaoPu Foundation of Fujian Medical University (No. 2015MP037).

Conwell, S. E., White, A. E., Harper, J. W., and Knipe, D. M. (2015). Identification of TRIM27 as a novel degradation target of herpes simplex virus 1 ICP0. J. Virol. 89, 220-229. doi: 10.1128/JVI.02635-14

da Silva, L. F., and Jones, C. (2013). Small non-coding RNAs encoded within the herpes simplex virus type 1 latency associated transcript (LAT) cooperate with the retinoic acid inducible gene I (RIG-I) to induce beta-interferon promoter activity and promote cell survival. Virus Res. 175, 101-109. doi: 10.1016/j.virusres.2013.04.005

Dansako, H., Ueda, Y., Okumura, N., Satoh, S., Sugiyama, M., Mizokami, M., et al. (2016). The cyclic GMP-AMP synthetase-STING signaling pathway is required for both the innate immune response against HBV and the suppression of HBV assembly. FEBS J. 283, 144-156. doi: 10.1111/ febs. 13563

Davison, A. J. (2010). Herpesvirus systematics. Vet. Microbiol. 143, 52-69. doi: 10.1016/j.vetmic.2010.02.014

Decker, T., Muller, M., and Stockinger, S. (2005). The yin and yang of type I interferon activity in bacterial infection. Nat. Rev. Immunol. 5, 675-687. doi: $10.1038 /$ nri1684

Deretic, V., Saitoh, T., and Akira, S. (2013). Autophagy in infection, inflammation and immunity. Nat. Rev. Immunol. 13, 722-737. doi: 10.1038/nri3532

Deschamps, T., and Kalamvoki, M. (2017). Evasion of the STING DNA-sensing pathway by VP11/12 of herpes simplex virus 1. J. Virol. 91. doi: 10.1128/ JVI.00535-17

Devanand, D. P. (2018). Viral hypothesis and antiviral treatment in Alzheimer's disease. Curr. Neurol. Neurosci. Rep. 18:55. doi: 10.1007/s11910-018-0863-1

Dong, X., and Levine, B. (2013). Autophagy and viruses: adversaries or allies? J. Innate Immun. 5, 480-493. doi: 10.1159/000346388

Farooq, A. V., and Shukla, D. (2012). Herpes simplex epithelial and stromal keratitis: an epidemiologic update. Surv. Ophthalmol. 57, 448-462. doi: 10.1016/j.survophthal.2012.01.005

Gao, D., Wu, J., Wu, Y. T., Du, F., Aroh, C., Yan, N., et al. (2013). Cyclic GMP-AMP synthase is an innate immune sensor of HIV and other retroviruses. Science 341, 903-906. doi: 10.1126/science.1240933

Hayden, M. S., and Ghosh, S. (2008). Shared principles in NF-kappaB signaling. Cell 132, 344-362. doi: 10.1016/j.cell.2008.01.020

Henderson, G., Peng, W., Jin, L., Perng, G. C., Nesburn, A. B., Wechsler, S. L., et al. (2002). Regulation of caspase 8- and caspase 9-induced apoptosis by the herpes simplex virus type 1 latency-associated transcript. J. Neurovirol. 8(Suppl. 2), 103-111. doi: 10.1080/13550280290101085 
Horowitz, R., Aierstuck, S., Williams, E. A., and Melby, B. (2010). Herpes simplex virus infection in a university health population: clinical manifestations, epidemiology, and implications. J. Am. Coll. Heal. 59, 69-74. doi: 10.1080/07448481.2010.483711

Huang, J., You, H., Su, C., Li, Y., Chen, S., and Zheng, C. (2018). Herpes simplex virus 1 tegument protein VP22 abrogates cGAS/STING-mediated antiviral innate immunity. J. Virol. 92. doi: 10.1128/JVI.00841-18

Ibanez, F. J., Farias, M. A., Gonzalez-Troncoso, M. P., Corrales, N., Duarte, L. F., Retamal-Diaz, A., et al. (2018). Experimental dissection of the lytic replication cycles of herpes simplex viruses in vitro. Front. Microbiol. 9:2406. doi: $10.3389 /$ fmicb.2018.02406

Ishikawa, H., and Barber, G. N. (2011). The STING pathway and regulation of innate immune signaling in response to DNA pathogens. Cell. Mol. Life Sci. 68, 1157-1165. doi: 10.1007/s00018-010-0605-2

Ivashkiv, L. B., and Donlin, L. T. (2014). Regulation of type I interferon responses. Nat. Rev. Immunol. 14, 36-49. doi: 10.1038/nri3581

Iversen, M. B., Ank, N., Melchjorsen, J., and Paludan, S. R. (2010). Expression of type III interferon (IFN) in the vaginal mucosa is mediated primarily by dendritic cells and displays stronger dependence on NF-kappaB than type I IFNs. J. Virol. 84, 4579-4586. doi: 10.1128/JVI.02591-09

Iwai, K. (2014). Diverse roles of the ubiquitin system in NF-kappaB activation. Biochim. Biophys. Acta 1843, 129-136. doi: 10.1016/j.bbamcr.2013.03.011

Iwasaki, A. (2012). A virological view of innate immune recognition. Annu. Rev. Microbiol. 66, 177-196. doi: 10.1146/annurev-micro-092611-150203

Jiang, Z., Su, C., and Zheng, C. (2016). Herpes simplex virus 1 tegument protein UL41 counteracts IFIT3 antiviral innate immunity. J. Virol. 90, 11056-11061. doi: 10.1128/JVI.01672-16

Johnson, K. E., Chikoti, L., and Chandran, B. (2013). Herpes simplex virus 1 infection induces activation and subsequent inhibition of the IFI16 and NLRP3 inflammasomes. J. Virol. 87, 5005-5018. doi: 10.1128/JVI.00082-13

Johnson, K. E., and Knipe, D. M. (2010). Herpes simplex virus-1 infection causes the secretion of a type I interferon-antagonizing protein and inhibits signaling at or before Jak-1 activation. Virology 396, 21-29. doi: 10.1016/j. virol.2009.09.021

Johnson, K. E., Song, B., and Knipe, D. M. (2008). Role for herpes simplex virus 1 ICP27 in the inhibition of type I interferon signaling. Virology 374, 487-494. doi: 10.1016/j.virol.2008.01.001

Jurak, I., Silverstein, L. B., Sharma, M., and Coen, D. M. (2012). Herpes simplex virus is equipped with RNA- and protein-based mechanisms to repress expression of ATRX, an effector of intrinsic immunity. J. Virol. 86, 10093-10102. doi: 10.1128/JVI.00930-12

Kim, J. C., Lee, S. Y., Kim, S. Y., Kim, J. K., Kim, H. J., Lee, H. M., et al. (2008). HSV-1 ICP27 suppresses NF-kappaB activity by stabilizing IkappaBalpha. FEBS Lett. 582, 2371-2376. doi: 10.1016/j.febslet.2008.05.044

Koelle, D. M., Norberg, P., Fitzgibbon, M. P., Russell, R. M., Greninger, A. L., Huang, M. L., et al. (2017). Worldwide circulation of HSV-2 x HSV-1 recombinant strains. Sci. Rep. 7:44084. doi: 10.1038/srep44084

Levine, B., Mizushima, N., and Virgin, H. W. (2011). Autophagy in immunity and inflammation. Nature 469, 323-335. doi: 10.1038/nature09782

Li, S., Kong, L., and Yu, X. (2015). The expanding roles of endoplasmic reticulum stress in virus replication and pathogenesis. Crit. Rev. Microbiol. 41, 150-164. doi: 10.3109/1040841X.2013.813899

Lilley, C. E., Chaurushiya, M. S., Boutell, C., Landry, S., Suh, J., Panier, S., et al. (2010). A viral E3 ligase targets RNF8 and RNF168 to control histone ubiquitination and DNA damage responses. EMBO J. 29, 943-955. doi: 10.1038/ emboj.2009.400

Lima, G. K., Zolini, G. P., Mansur, D. S., Freire Lima, B. H., Wischhoff, U., Astigarraga, R. G., et al. (2010). Toll-like receptor (TLR) 2 and TLR9 expressed in trigeminal ganglia are critical to viral control during herpes simplex virus 1 infection. Am. J. Pathol. 177, 2433-2445. doi: 10.2353/ ajpath.2010.100121

Liu, X., Main, D., Ma, Y., and He, B. (2018). Herpes simplex virus 1 inhibits TANK-binding kinase 1 through formation of the Us11-Hsp90 complex. J. Virol. 92. doi: 10.1128/JVI.00402-18

Liu, J., Qian, C., and Cao, X. (2016). Post-translational modification control of innate immunity. Immunity 45, 15-30. doi: 10.1016/j.immuni.2016.06.020

Liu, X., Wang, Q., Chen, W., and Wang, C. (2013). Dynamic regulation of innate immunity by ubiquitin and ubiquitin-like proteins. Cytokine Growth Factor Rev. 24, 559-570. doi: 10.1016/j.cytogfr.2013.07.002
Lugrin, J., and Martinon, F. (2018). The AIM2 inflammasome: sensor of pathogens and cellular perturbations. Immunol. Rev. 281, 99-114. doi: 10.1111/imr.12618

Lukashchuk, V., and Everett, R. D. (2010). Regulation of ICP0-null mutant herpes simplex virus type 1 infection by ND10 components ATRX and hDaxx. J. Virol. 84, 4026-4040. doi: 10.1128/JVI.02597-09

Lund, J., Sato, A., Akira, S., Medzhitov, R., and Iwasaki, A. (2003). Toll-like receptor 9-mediated recognition of herpes simplex virus- 2 by plasmacytoid dendritic cells. J. Exp. Med. 198, 513-520. doi: 10.1084/jem.20030162

Lussignol, M., and Esclatine, A. (2017). Herpesvirus and autophagy: "all right, everybody be cool, this is a robbery!”. Viruses 9. doi: 10.3390/v9120372

Ma, Z., Jacobs, S. R., West, J. A., Stopford, C., Zhang, Z., Davis, Z., et al. (2015). Modulation of the cGAS-STING DNA sensing pathway by gammaherpesviruses. Proc. Natl. Acad. Sci. USA 112, E4306-E4315. doi: 10.1073/pnas.1503831112

Mandell, M. A., Jain, A., Arko-Mensah, J., Chauhan, S., Kimura, T., Dinkins, C., et al. (2014). TRIM proteins regulate autophagy and can target autophagic substrates by direct recognition. Dev. Cell 30, 394-409. doi: 10.1016/j. devcel.2014.06.013

Maruzuru, Y., Ichinohe, T., Sato, R., Miyake, K., Okano, T., Suzuki, T., et al. (2018). Herpes simplex virus 1 VP22 inhibits AIM2-dependent inflammasome activation to enable efficient viral replication. Cell Host Microbe 23, 254-265 e257. doi: 10.1016/j.chom.2017.12.014

Merkl, P. E., and Knipe, D. M. (2019). Role for a filamentous nuclear assembly of IFI16, DNA, and host factors in restriction of herpesviral infection. MBio 10. doi: $10.1128 / \mathrm{mBio} .02621-18$

Merkl, P. E., Orzalli, M. H., and Knipe, D. M. (2018). Mechanisms of host IFI16, PML, and Daxx protein restriction of herpes simplex virus 1 replication. J. Virol. 92. doi: 10.1128/JVI.00057-18

Orzalli, M. H., Broekema, N. M., and Knipe, D. M. (2016). Relative contributions of herpes simplex virus 1 ICP0 and vhs to loss of cellular IFI16 vary in different human cell types. J. Virol. 90, 8351-8359. doi: 10.1128/ JVI.00939-16

Orzalli, M. H., Conwell, S. E., Berrios, C., DeCaprio, J. A., and Knipe, D. M. (2013). Nuclear interferon-inducible protein 16 promotes silencing of herpesviral and transfected DNA. Proc. Natl. Acad. Sci. USA 110, E4492-E4501. doi: 10.1073/pnas.1316194110

Orzalli, M. H., DeLuca, N. A., and Knipe, D. M. (2012). Nuclear IFI16 induction of IRF-3 signaling during herpesviral infection and degradation of IFI16 by the viral ICP0 protein. Proc. Natl. Acad. Sci. USA 109, E3008-E3017. doi: $10.1073 /$ pnas.1211302109

Orzalli, M. H., and Knipe, D. M. (2014). Cellular sensing of viral DNA and viral evasion mechanisms. Annu. Rev. Microbiol. 68, 477-492. doi: 10.1146/ annurev-micro-091313-103409

Paijo, J., Doring, M., Spanier, J., Grabski, E., Nooruzzaman, M., Schmidt, T., et al. (2016). cGAS senses human cytomegalovirus and induces type I interferon responses in human monocyte-derived cells. PLoS Pathog. 12:e1005546. doi: 10.1371/journal.ppat.1005546

Perng, G. C., Jones, C., Ciacci-Zanella, J., Stone, M., Henderson, G., Yukht, A., et al. (2000). Virus-induced neuronal apoptosis blocked by the herpes simplex virus latency-associated transcript. Science 287, 1500-1503. doi: 10.1126/ science.287.5457.1500

Phelan, D., Barrozo, E. R., and Bloom, D. C. (2017). HSV1 latent transcription and non-coding RNA: a critical retrospective. J. Neuroimmunol. 308, 65-101. doi: 10.1016/j.jneuroim.2017.03.002

Pilli, M., Arko-Mensah, J., Ponpuak, M., Roberts, E., Master, S., Mandell, M. A., et al. (2012). TBK-1 promotes autophagy-mediated antimicrobial defense by controlling autophagosome maturation. Immunity 37, 223-234. doi: 10.1016/j.immuni.2012.04.015

Prabakaran, T., Bodda, C., Krapp, C., Zhang, B. C., Christensen, M. H., Sun, C., et al. (2018). Attenuation of cGAS-STING signaling is mediated by a p62/SQSTM1-dependent autophagy pathway activated by TBK1. EMBO J. 37. doi: $10.15252 / \mathrm{embj} .201797858$

Raftery, N., and Stevenson, N. J. (2017). Advances in anti-viral immune defence: revealing the importance of the IFN JAK/STAT pathway. Cell. Mol. Life Sci. 74, 2525-2535. doi: 10.1007/s00018-017-2520-2

Richter, B., Sliter, D. A., Herhaus, L., Stolz, A., Wang, C., Beli, P., et al. (2016). Phosphorylation of OPTN by TBK1 enhances its binding to Ub chains and promotes selective autophagy of damaged mitochondria. Proc. Natl. Acad. Sci. USA 113, 4039-4044. doi: 10.1073/pnas.1523926113 
Saitoh, T., Fujita, N., Hayashi, T., Takahara, K., Satoh, T., Lee, H., et al. (2009). Atg9a controls dsDNA-driven dynamic translocation of STING and the innate immune response. Proc. Natl. Acad. Sci. USA 106, 20842-20846. doi: 10.1073/pnas.0911267106

Sandri-Goldin, R. M. (2011). The many roles of the highly interactive HSV protein ICP27, a key regulator of infection. Future Microbiol. 6, 1261-1277. doi: $10.2217 / \mathrm{fmb} .11 .119$

Schneider, W. M., Chevillotte, M. D., and Rice, C. M. (2014). Interferonstimulated genes: a complex web of host defenses. Annu. Rev. Immunol. 32, 513-545. doi: 10.1146/annurev-immunol-032713-120231

Schoggins, J. W. (2019). Interferon-stimulated genes: what do they all do? Annu. Rev. Virol. 6, 567-584. doi: 10.1146/annurev-virology-092818-015756

Schoggins, J. W., and Rice, C. M. (2011). Interferon-stimulated genes and their antiviral effector functions. Curr. Opin. Virol. 1, 519-525. doi: 10.1016/j. coviro.2011.10.008

Schulz, K. S., and Mossman, K. L. (2016). Viral evasion strategies in type I IFN signaling - a summary of recent developments. Front. Immunol. 7:498. doi: $10.3389 /$ fimmu.2016.00498

Sharma, V., Mobeen, F., and Prakash, T. (2016). Comparative genomics of Herpesviridae family to look for potential signatures of human infecting strains. Int. J. Genomics 2016:9543274. doi: 10.1155/2016/9543274

Sharma, S., tenOever, B. R., Grandvaux, N., Zhou, G. P., Lin, R., and Hiscott, J. (2003). Triggering the interferon antiviral response through an IKK-related pathway. Science 300, 1148-1151. doi: 10.1126/science.1081315

Shen, G., Wang, K., Wang, S., Cai, M., Li, M. L., and Zheng, C. (2014). Herpes simplex virus 1 counteracts viperin via its virion host shutoff protein UL41. J. Virol. 88, 12163-12166. doi: 10.1128/JVI.01380-14

Sparrer, K. M. J., Gableske, S., Zurenski, M. A., Parker, Z. M., Full, F., Baumgart, G. J., et al. (2017). TRIM23 mediates virus-induced autophagy via activation of TBK1. Nat. Microbiol. 2, 1543-1557. doi: 10.1038/s41564-017-0017-2

Stempel, M., Chan, B., and Brinkmann, M. M. (2019). Coevolution pays off: Herpesviruses have the license to escape the DNA sensing pathway. Med. Microbiol. Immunol. 208, 495-512. doi: 10.1007/s00430-019-00582-0

Su, C., Zhan, G., and Zheng, C. (2016). Evasion of host antiviral innate immunity by HSV-1, an update. Virol. J. 13:38. doi: 10.1186/s12985-016-0495-5

Su, C., Zhang, J., and Zheng, C. (2015). Herpes simplex virus 1 UL41 protein abrogates the antiviral activity of hZAP by degrading its mRNA. Virol. J. 12:203. doi: 10.1186/s12985-015-0433-y

Su, C., and Zheng, C. (2017). Herpes simplex virus 1 abrogates the cGAS/ STING-mediated cytosolic DNA-sensing pathway via its virion host shutoff protein, UL41. J. Virol. 91. doi: 10.1128/JVI.02414-16

Sun, C., Schattgen, S. A., Pisitkun, P., Jorgensen, J. P., Hilterbrand, A. T., Wang, L. J., et al. (2015). Evasion of innate cytosolic DNA sensing by a gammaherpesvirus facilitates establishment of latent infection. J. Immunol. 194, 1819-1831. doi: 10.4049/jimmunol.1402495

Sun, L., Wu, J., Du, F., Chen, X., and Chen, Z. J. (2013). Cyclic GMP-AMP synthase is a cytosolic DNA sensor that activates the type I interferon pathway. Science 339, 786-791. doi: 10.1126/science.1232458

Tal, M. C., and Iwasaki, A. (2009). Autophagy and innate recognition systems. Curr. Top. Microbiol. Immunol. 335, 107-121. doi: 10.1007/978-3-642-00302-8_5

Tognarelli, E. I., Palomino, T. F., Corrales, N., Bueno, S. M., Kalergis, A. M., and Gonzalez, P. A. (2019). Herpes simplex virus evasion of early host antiviral responses. Front. Cell. Infect. Microbiol. 9:127. doi: 10.3389/ fcimb.2019.00127

Tormanen, K., Allen, S., Mott, K. R., and Ghiasi, H. (2019). The latencyassociated transcript inhibits apoptosis via downregulation of components of the type I interferon pathway during latent herpes simplex virus 1 ocular infection. J. Virol. 93. doi: 10.1128/JVI.00103-19

Tsuchida, T., Zou, J., Saitoh, T., Kumar, H., Abe, T., Matsuura, Y., et al. (2010). The ubiquitin ligase TRIM56 regulates innate immune responses to intracellular double-stranded DNA. Immunity 33, 765-776. doi: 10.1016/j.immuni. 2010.10.013

Unterholzner, L., Keating, S. E., Baran, M., Horan, K. A., Jensen, S. B., Sharma, S., et al. (2010). IFI16 is an innate immune sensor for intracellular DNA. Nat. Immunol. 11, 997-1004. doi: 10.1038/ni.1932

Uyangaa, E., Choi, J. Y., Patil, A. M., Hossain, F. M. A., Park, S. O., Kim, B., et al. (2018). Dual TLR2/9 recognition of herpes simplex virus infection is required for recruitment and activation of monocytes and NK cells and restriction of viral dissemination to the central nervous system. Front. Immunol. 9:905. doi: 10.3389/fimmu.2018.00905

van Pesch, V., Lanaya, H., Renauld, J. C., and Michiels, T. (2004). Characterization of the murine alpha interferon gene family. J. Virol. 78, 8219-8228. doi: 10.1128/JVI.78.15.8219-8228.2004

Versteeg, G. A., Rajsbaum, R., Sanchez-Aparicio, M. T., Maestre, A. M., Valdiviezo, J., Shi, M., et al. (2013). The E3-ligase TRIM family of proteins regulates signaling pathways triggered by innate immune pattern-recognition receptors. Immunity 38, 384-398. doi: 10.1016/j.immuni.2012.11.013

Wang, K., Ni, L., Wang, S., and Zheng, C. (2014). Herpes simplex virus 1 protein kinase US3 hyperphosphorylates p65/RelA and dampens NF-kappaB activation. J. Virol. 88, 7941-7951. doi: 10.1128/JVI.03394-13

Wang, S., Wang, K., Lin, R., and Zheng, C. (2013). Herpes simplex virus 1 serine/threonine kinase US3 hyperphosphorylates IRF3 and inhibits beta interferon production. J. Virol. 87, 12814-12827. doi: 10.1128/ JVI.02355-13

Wang, L., Wen, M., and Cao, X. (2019). Nuclear hnRNPA2B1 initiates and amplifies the innate immune response to DNA viruses. Science 365. doi: 10.1126/science.aav0758

Woronicz, J. D., Gao, X., Cao, Z., Rothe, M., and Goeddel, D. V. (1997). IkappaB kinase-beta: NF-kappaB activation and complex formation with IkappaB kinase-alpha and NIK. Science 278, 866-869. doi: 10.1126/science.278.5339.866

$\mathrm{Wu}, \mathrm{J}$., and Chen, Z. J. (2014). Innate immune sensing and signaling of cytosolic nucleic acids. Annu. Rev. Immunol. 32, 461-488. doi: 10.1146/annurevimmunol-032713-120156

Wu, J. J., Li, W., Shao, Y., Avey, D., Fu, B., Gillen, J., et al. (2015). Inhibition of cGAS DNA sensing by a herpesvirus virion protein. Cell Host Microbe 18, 333-344. doi: 10.1016/j.chom.2015.07.015

Wu, W., Newcomb, W. W., Cheng, N., Aksyuk, A., Winkler, D. C., and Steven, A. C. (2016). Internal proteins of the procapsid and mature capsids of herpes simplex virus 1 mapped by bubblegram imaging. J. Virol. 90, 5176-5186. doi: 10.1128/JVI.03224-15

Wu, J., Sun, L., Chen, X., Du, F., Shi, H., Chen, C., et al. (2013). Cyclic GMP-AMP is an endogenous second messenger in innate immune signaling by cytosolic DNA. Science 339, 826-830. doi: 10.1126/science.1229963

Xing, J., Ni, L., Wang, S., Wang, K., Lin, R., and Zheng, C. (2013). Herpes simplex virus 1-encoded tegument protein VP16 abrogates the production of beta interferon (IFN) by inhibiting NF-kappaB activation and blocking IFN regulatory factor 3 to recruit its coactivator CBP. J. Virol. 87, 9788-9801. doi: 10.1128/JVI.01440-13

Xing, J., Zhang, A., Zhang, H., Wang, J., Li, X. C., Zeng, M. S., et al. (2017). TRIM29 promotes DNA virus infections by inhibiting innate immune response. Nat. Commun. 8, 945. doi: 10.1038/s41467-017-00101-w

Xu, H., Su, C., Pearson, A., Mody, C. H., and Zheng, C. (2017). Herpes simplex virus 1 UL24 abrogates the DNA sensing signal pathway by inhibiting NFkappaB activation. J. Virol. 91. doi: 10.1128/JVI.00025-17

Ye, R., Su, C., Xu, H., and Zheng, C. (2017). Herpes simplex virus 1 ubiquitinspecific protease UL36 abrogates NF-kappaB activation in DNA sensing signal pathway. J. Virol. 91. doi: 10.1128/JVI.02417-16

You, H., Yuan, H., Fu, W., Su, C., Wang, W., Cheng, T., et al. (2017). Herpes simplex virus type 1 abrogates the antiviral activity of $\mathrm{Ch} 25 \mathrm{~h}$ via its virion host shutoff protein. Antivir. Res. 143, 69-73. doi: 10.1016/j. antiviral.2017.04.004

You, H., Zheng, S., Huang, Z., Lin, Y., Shen, Q., and Zheng, C. (2019). Herpes simplex virus 1 tegument protein UL46 inhibits TANK-binding kinase 1-mediated signaling. MBio 10. doi: 10.1128/mBio.00919-19

Yuan, H., You, J., You, H., and Zheng, C. (2018). Herpes simplex virus 1 UL36USP antagonizes type I interferon-mediated antiviral innate immunity. J. Virol. 92. doi: 10.1128/JVI.01161-18

Zenner, H. L., Mauricio, R., Banting, G., and Crump, C. M. (2013). Herpes simplex virus 1 counteracts tetherin restriction via its virion host shutoff activity. J. Virol. 87, 13115-13123. doi: 10.1128/JVI.02167-13

Zhang, J., Hu, M. M., Wang, Y. Y., and Shu, H. B. (2012). TRIM32 protein modulates type I interferon induction and cellular antiviral response by targeting MITA/STING protein for K63-linked ubiquitination. J. Biol. Chem. 287, 28646-28655. doi: 10.1074/jbc.M112.362608

Zhang, D., Su, C., and Zheng, C. (2016). Herpes simplex virus 1 serine protease VP24 blocks the DNA-sensing signal pathway by abrogating 
activation of interferon regulatory factor 3. J. Virol. 90, 5824-5829. doi: 10.1128/JVI.00186-16

Zhang, J., Wang, K., Wang, S., and Zheng, C. (2013a). Herpes simplex virus 1 E3 ubiquitin ligase ICP0 protein inhibits tumor necrosis factor alphainduced NF-kappaB activation by interacting with p65/RelA and p50/NFkappaB1. J. Virol. 87, 12935-12948. doi: 10.1128/JVI.01952-13

Zhang, J., Wang, S., Wang, K., and Zheng, C. (2013b). Herpes simplex virus 1 DNA polymerase processivity factor UL42 inhibits TNF-alpha-induced NF-kappaB activation by interacting with p65/RelA and p50/NF-kappaB1. Med. Microbiol. Immunol. 202, 313-325. doi: 10.1007/s00430-013-0295-0

Zhang, Z., Yuan, B., Bao, M., Lu, N., Kim, T., and Liu, Y. J. (2011). The helicase DDX41 senses intracellular DNA mediated by the adaptor STING in dendritic cells. Nat. Immunol. 12, 959-965. doi: 10.1038/ni.2091

Zhang, J., Zhao, J., Xu, S., Li, J., He, S., Zeng, Y., et al. (2018). Species-specific Deamidation of cGAS by herpes simplex virus UL37 protein facilitates viral replication. Cell Host Microbe 24, 234-248 e235. doi: 10.1016/j.chom.2018.07.004
Zheng, C. (2018). Evasion of cytosolic DNA-stimulated innate immune responses by herpes simplex virus 1. J. Virol. 92. doi: 10.1128/JVI.00099-17

Zhou, L., Li, J., Wang, X., Ye, L., Hou, W., Ho, J., et al. (2011). IL-29/IL-28A suppress HSV-1 infection of human NT2-N neurons. J. Neurovirol. 17, 212-219. doi: 10.1007/s13365-011-0031-8

Conflict of Interest: The authors declare that the research was conducted in the absence of any commercial or financial relationships that could be construed as a potential conflict of interest.

Copyright (c) 2019 Lin and Zheng. This is an open-access article distributed under the terms of the Creative Commons Attribution License (CC BY). The use, distribution or reproduction in other forums is permitted, provided the original author(s) and the copyright owner(s) are credited and that the original publication in this journal is cited, in accordance with accepted academic practice. No use, distribution or reproduction is permitted which does not comply with these terms. 